\title{
Process Management Framework: Guidance to Successful Implementation of Processes in Clinical Development
}

Therapeutic Innovation \& Regulatory Science 2019, Vol. 53(I) 25-35 (c) The Author(s) 2019 Article reuse guidelines: sagepub.com/journals-permissions DOI: $10.1177 / 216847901881775$ | tirs.sagepub.com

\author{
Michael Edward Husovich, BS $^{\prime}{ }^{\mathbb{D}}$, Ruzica Zadro, $\mathbf{M D}^{2}$, \\ Lora Lee Zoller-Neuner, BSN ${ }^{3}$, Griet Vangheel, $\mathbf{M S}^{4}$, \\ Odette Anyangwe, RN, BSc, MPH, MSC ${ }^{5}$, Diane Puglia Ryan, MS $^{6}$, \\ and Beata Rygiel-Zbikowska, MD, $\mathrm{MBA}^{7}$
}

\begin{abstract}
Clearly defined, documented, and managed processes form the foundation for how we effectively develop medicines for our patients. For this reason, process has been identified as a primary "element" of an effective quality management system (QMS) as described in the TransCelerate clinical quality management system (CQMS) conceptual framework. The importance of identifying and effectively managing processes is also emphasized in ICH GCP E6 (R2) in the new Section 5.0 Quality Management. An effective process management framework is fundamental to ensure the efficient and effective delivery of clinical development programs, enhance quality and productivity, and ultimately benefit our ability to deliver needed treatments to patients. The aim of this paper is to provide a conceptual process management framework to be used as guidance for effective process mapping, process documentation, implementation of optimal learning methods, and ensuring ongoing process performance evaluation and continuous improvement.
\end{abstract}

\section{Keywords}

TransCelerate, QMS, clinical development, process management, end to-end process

\section{Introduction}

Development and delivery of medicines on a global scale has presented great benefit to patients; however, it has also added complexity to our business and the processes by which we develop our medicines. The need for clear and consistent processes, associated documentation, and training to ensure staff effectively follow these processes is becoming increasingly important as companies search for effective and efficient ways to deliver medicines worldwide while ensuring the protection and safety of our patients.

The TransCelerate clinical quality management system (CQMS) conceptual framework ${ }^{1}$ highlighted process as a primary and integral element of a CQMS. The intent of this paper is to define a conceptual framework for implementing processes in clinical development. Prior to discussing the framework for implementing processes, a few key process management concepts need to be considered. (Note: A process "implementation toolkit" has been developed that provides additional tools and working examples to further aid in implementation of the concepts and framework discussed in this manuscript. These tools and examples will be available on the TransCelerate webpage at www.transceleratebiopharmainc.com.)

\section{Process Management Key Concepts}

Process management is a fundamental shift from a traditional (function-driven) approach to an end-to-end (E2E) process (customer-driven) approach (Figure 1).

In a traditional organizational approach, organizations are organized and measured by departmental goals and objectives. Executive leaders operate as a team, but their energy and focus are directed toward their functions.

\footnotetext{
' Global Quality, Amgen, Thousand Oaks, CA, USA

${ }^{2}$ Quality \& Continuous Improvement, Global Clinical Trial Operations, Merck Sharp \& Dohme, Zagreb, Croatia

${ }^{3}$ Clinical Quality \& Continuous Improvement, Sanofi, Bridgewater, New Jersey, USA

${ }^{4}$ BioResearch Quality and Compliance, Johnson \& Johnson, Beerse, Belgium

${ }^{5}$ Pharma Development Quality (PDQ), Roche, Basel, Switzerland

${ }^{6}$ Clinical Development Quality - Process Quality, Pfizer, Groton, CT, USA

${ }^{7}$ Clinical Operations, GMD, AstraZeneca, Warszawa, Poland
}

Submitted 0I-Jun-2018; accepted 03-Oct-2018

Corresponding Author:

Michael Edward Husovich, BS, Global Quality, Amgen, I Amgen Center Drive, Thousand Oaks, CA 91320, USA.

Email: husovich@amgen.com 


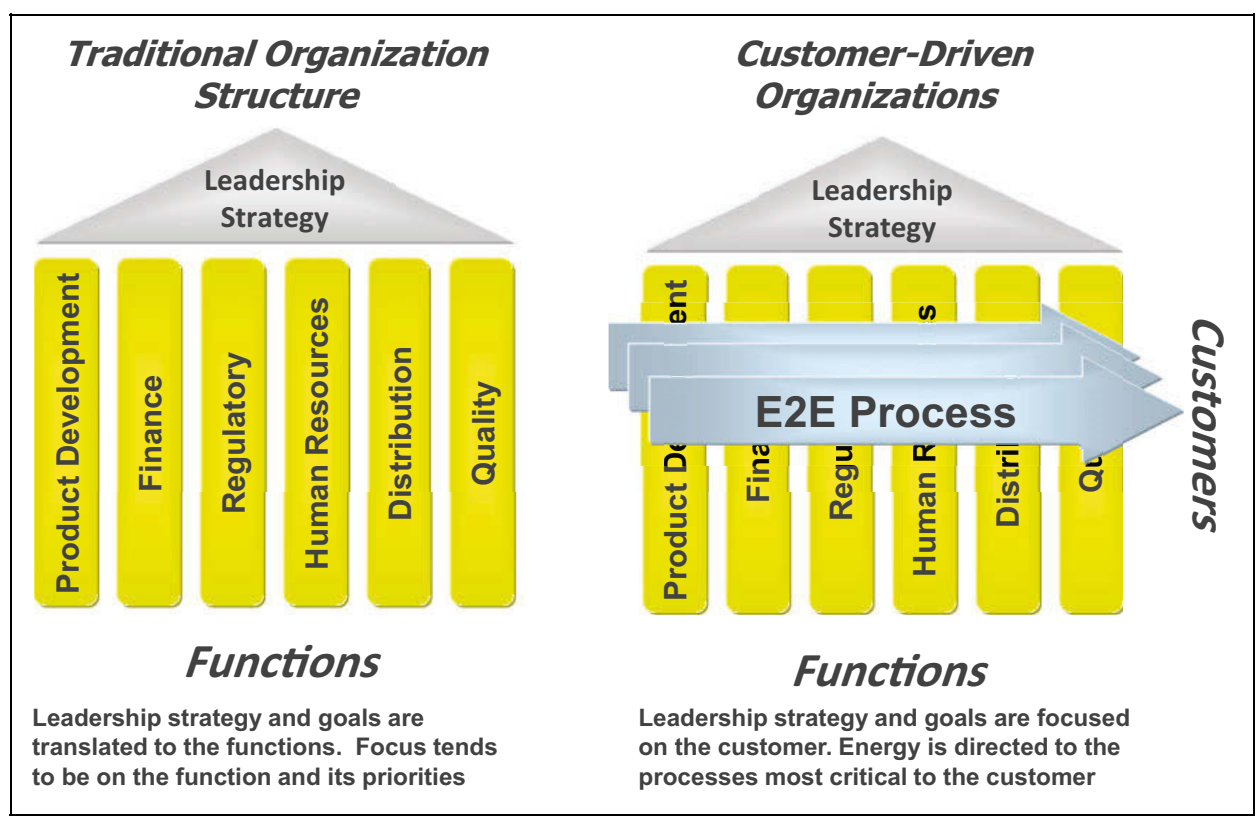

Figure I. Traditional versus customer-driven approach.

In a customer-driven approach, the organizational focus is on the customer. Critical processes important to the customer are readily identified and focused on. Executive leaders realize that products and services for customers are not produced by functions but by processes that span end-to-end across functions. Therefore, processes need to be simple, robust, and compliant. Functions are still important as they own the necessary skills and competencies to carry out the processes.

It is important to note that the patient is the ultimate "external" customer of clinical development as we develop medicines to serve patients. Health authorities who approve medicines are also considered key "external" customers as we produce clinical data and submit it for their review. However, there are also important "internal" customers of processes (eg, clinical scientists who assess clinical data, quality managers who assess quality and compliance of how clinical data are produced, or operational managers who assess process efficiency). Understanding the steps to execute clinical development processes and driving process excellence now becomes leverage to serve "external and internal customers" as effectively as possible. We believe the process or customer-driven approach offers the following advantages:

$\checkmark$ Accountability: Focuses on overcoming functional area silos through single points of process accountability.

$\checkmark$ Quality: Clearly defined processes, documentation, and training enable "right the first time" operations, improving quality and reducing risk.

$\checkmark$ Compliance: Clearly defined processes give confidence to health authorities on sponsors' intentions of meeting regulatory requirements.

$\checkmark \quad$ Speed: Provides end-to-end performance management and "sightline" to customer.
While most of the above concepts of process management or a process-driven approach are easy to understand, they are often difficult to apply. In May 2017, a voluntary anonymous survey of 19 TransCelerate member companies indicated that $80 \%$ of member companies have started or are well underway in the implementation of a process-driven approach. However, member companies cited challenges associated with successful implementation. The primary challenges were (1) lack of leadership commitment, understanding, and change management and (2) lack of defined methodology or framework for implementing processes. Prior to beginning any large change initiative, such as moving from a function-driven approach to a process-driven approach, careful consideration should be given to effective leadership commitment, engagement, active participation, and advocacy of process management. While the lack of leadership commitment and change management cannot be underestimated, the primary intent of this paper is to provide a model process management framework that can be leveraged to successfully implement processes.

\section{Process Management Framework}

A process management framework ensures processes are identified, defined, and implemented in a common way to sustain and improve clinical development activities, quality objectives, and deliverables. This framework outlines a potential "roadmap" for implementing processes and allows for an optimal processes design to avoid gaps and overlaps. Figure 2 depicts a conceptual framework or methodology for process implementation. The steps described in the process management framework are applicable to organizations of all sizes; however, they may need to be scaled to accommodate the size of the organization. 


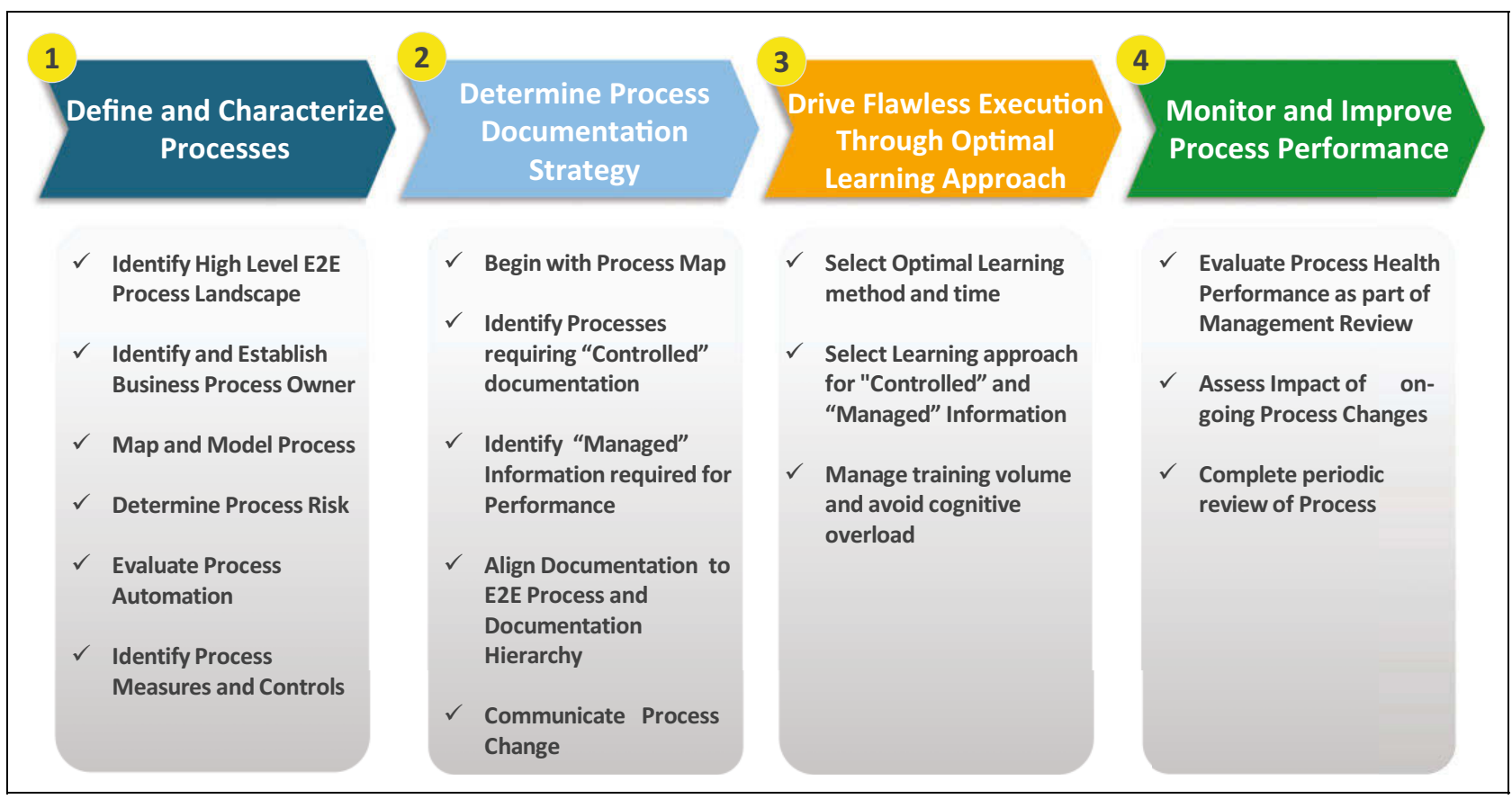

Figure 2. Process management framework. E2E, end to end.

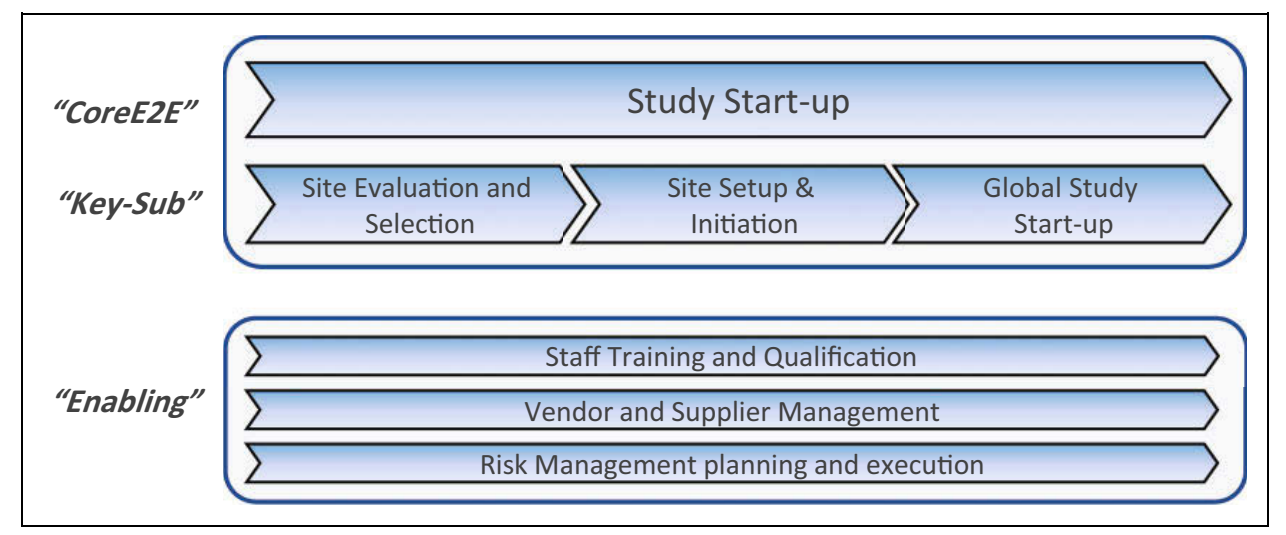

Figure 3. High-level E2E process landscape: study start-up. (This figure is for illustrative purposes only. Diagram is not intended to be a complete or recommended diagram for the subprocesses or enabling processes for study startup.)

\section{Define and Characterize Processes}

\section{Identify High-Level End-to-End (E2E) Process Landscape}

Clear process identification, ownership, and accountabilities are fundamental to ensure high-performing processes. The first step to implement processes is to understand the E2E process landscape. This is a fundamental and challenging first step as organizations move from a function-driven approach to a process-driven approach. One way to accomplish this is by identifying the "core" E2E processes, "sub" processes, and "enabling" processes within the clinical development area. A "core" process can be defined as a set of cross-functional valueadded activities or steps that deliver a specific output that has a profound impact on achieving strategic business objectives, either directly or indirectly. Key "sub-" processes constitute the sum of the core processes. Enabling processes do not necessarily belong to any one core or subprocess but are essential for the business to conduct its work. An example of core E2E, subprocesses, and enabling processes is illustrated in Figure 3 . The process of starting a clinical study can be considered a E2E "core" process, while site evaluation and selection, site setup and initiation, and global study startup are considered "subprocesses" and processes such as staff training and qualification, vendor and supplier management, and risk management meet the definition of "enabling" processes.

As additional "core" processes within an organization are identified, collectively these core processes should be depicted to represent the overall "E2E process view" or landscape for the organization. As companies mature on their journey toward process management, this depiction will become increasingly 
important to maintain organizational focus on processes while ensuring appropriate expertise and accountabilities are present in the functions to make the processes effective. (Note: The approach described above represents a "top down" approach. Alternatively, a "bottom-up approach" can be applied and has been proven effective. For instance, an internal audit or external inspection may have highlighted a process with deficiencies that require correction. Applying the processes framework on that particular process can be an effective way to demonstrate an example of "what good looks like" and help the organization on its journey towards a process-driven approach.)

\section{Identify and Establish Business Process Owner (BPO)}

The determination of which processes require a BPO is based on the relative importance and scope of the process. Generally, each core and subprocess should have a BPO. Using Figure 3 as an example, the recommendation is to have $4 \mathrm{BPOs}-1 \mathrm{BPO}$ to manage the E2E core process and 3 to manage the 3 subprocesses (1 BPO per subprocess). Because the BPOs play important roles, they should be carefully selected and adequately trained. Consideration should be given to leadership capability, domain expertise, empowerment, adequate resourcing, and ensuring they have the proper skills in process management for the related discipline. The goal of the BPO is to be a single point of ownership that drives process health and continuous improvement.

Typical responsibilities of the BPO include the following:

- Sets strategy and vision for the process

- Develops and manages policies and procedural documentation of the process

- Develops and improves the process through crossfunctional collaboration

- Monitors process performance through the development and review of key performance indicators and quality performance indicators (KPIs/QPIs)

- Communicates to senior leadership on process health and issues

Serves as the point of contact for process representation during regulatory inspections, internal audits, and/or discussions of systemic process quality issues

A BPO network or information-sharing forum is often useful for connecting processes, discussing cross-process issues, and driving collaboration across processes and functional areas.

\section{Map and Model Process}

Once the overall process landscape and BPOs are identified, individual process mapping and modeling can occur. Survey results indicated that $76 \%$ of TransCelerate member companies employ process-mapping and process-modeling techniques as precursors to the development of procedural documentation for clinical development. Effective process mapping is more than just a flow chart of activities. Effective process mapping and process modeling ensure that the process is defined correctly to articulate the desired business outcome or deliverable. Methodology for process mapping should be standardized to ensure consistency. Standard graphical notation such as business process modeling notation (BPMN) should be employed, and the modeling techniques should capture important data that can be used as part of an overall business process management framework. These data can include roles, interdependencies with other processes, inputs, outputs, systems, and documents. The quality-by-design principles of quality planning, control, and improvement, as well as general knowledge of metrics, risk management, and knowledge management, should also be used as part of the entire mapping exercise.

The methodology for process mapping may include the following activities:

- Assemble BPOs and process subject matter experts (SMEs)

- Ensure the key stakeholders who provide inputs or receive outputs of the process are engaged to provide or understand "customer" requirements for the process.

- Develop initial Suppliers, Inputs, Process, Outputs, and Customers (SIPOC) diagram. SIPOC is a process management tool from Six Sigma practices (Figure 4). This simple diagram begins the shift to process or customerdriven thinking. The goal of this map is to provide a high-level understanding of the process as it relates to the customer (anyone who received a deliverable or output from the process) and what the process requires as inputs (resources required by the process). Importantly, it also establishes the scope of the process. It helps to identify correctly key areas and stakeholders of the process to drive business deliverables and performance.

From the initial high-level SIPOC diagram, teams can create a more detailed process map. One type of process map that can be useful at this stage is a "swim lane" activity diagram. The goal of the swim lane activity diagram is to identify key milestones or activities, roles/responsibilities, handoffs, inputs/outputs, interdependencies, and associated information/ documents. A swim lane activity diagram is especially useful to depict roles and hand-offs that normally occur in the process. Identifying handoffs in the process is particularity useful as handoffs often represent opportunities for errors and inefficiencies. Figure 5 depicts a sample swim lane diagram for site setup activities between site selection and the site being initiated.

After teams have generated a swim lane diagram, they should ensure the diagram captures key roles, inputs, outputs, and interdependencies as well as other data including systems and documents. Collectively, these data can be referred to as "metadata." Metadata is data used to describe key characteristics of the process. These metadata, when used as part of an electronic business process management system, can enable powerful analyses to further define the interrelationships between processes. For example, in the swim lane diagram 


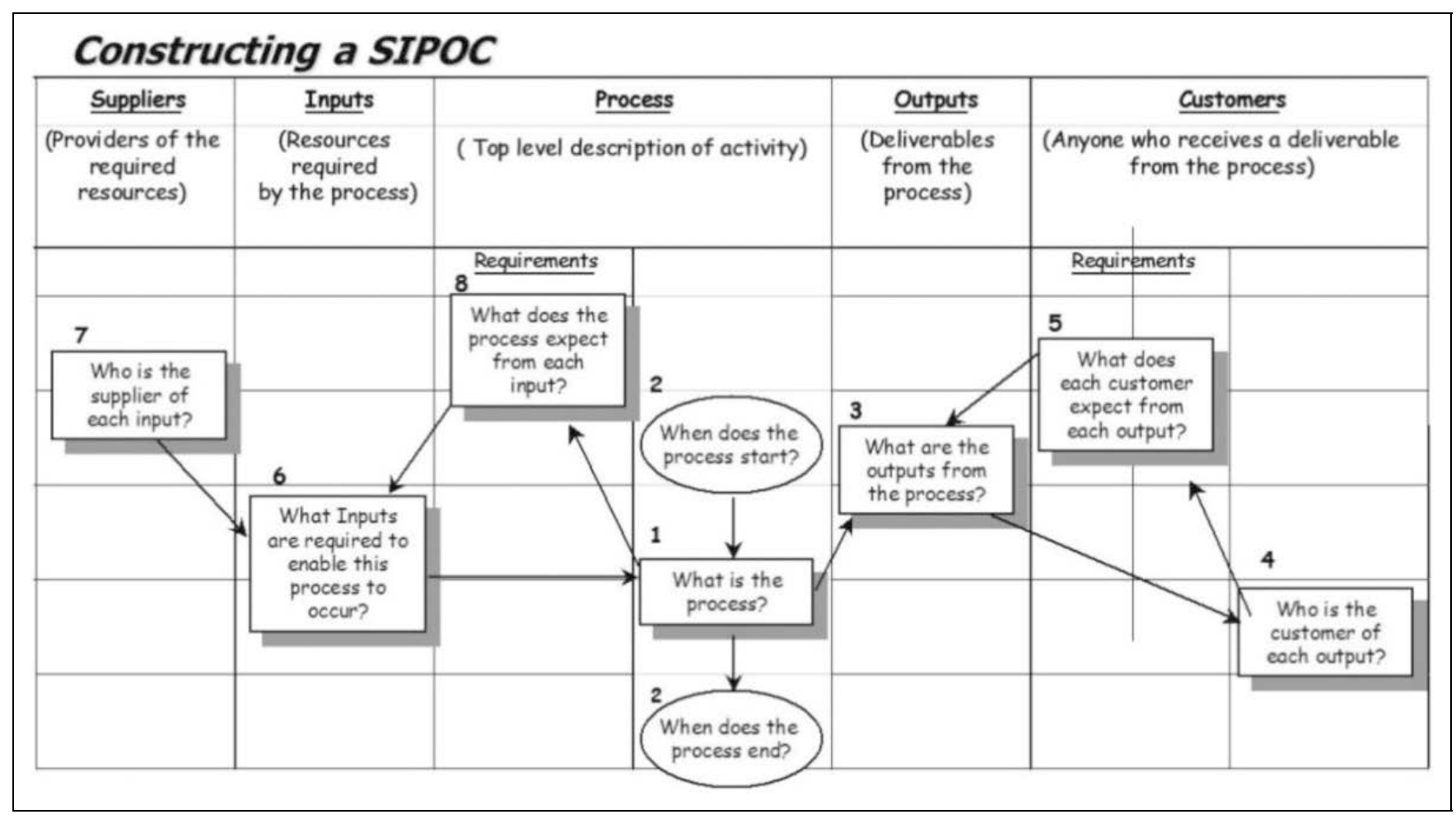

Figure 4. Example of a Suppliers, Inputs, Process, Outputs, and Customers (SIPOC) diagram.

example (Figure 5), the informed consent form (ICF) is captured as an input (metadata) into the "Create or Update Study Documents" step. By capturing the ICF as an input for this process along with other processes across the clinical development landscape, one can run a report from a business process management system to show where the ICF is used across all processes. Over time, this "impact analysis" becomes a powerful tool for effectively showing the impact of process changes or improvements as well as driving performance.

One way to simplify the identification of functional roles in documentation is to develop a generic roles master list. Each generic role is defined and includes information about which jobs may play that role. This information should be kept outside of the procedural document. The generic roles master list is used to identify roles (defined by a set of operational skills and competencies to conduct an activity or a set of operations) for a given process using common terminology across business entities, no matter what organization is in place. For example, a generic role can be clinician. Job titles of the person in the role of clinician include clinical scientist, clinical lead, and more. Generic roles provide flexibility to demonstrate when one or more job titles may be responsible for a role and longevity (ie, job titles may change over time, while generic roles will remain constant). After roles have been identified as part of process mapping, procedural documentation and training curricula can be assessed to determine impact to existing documentation and training.

\section{Determine Process Risk}

Processes in clinical development must be consistently characterized in terms of the risks to the protection of human subjects, data integrity/reliability of trial results, and meeting quality objectives. Specific to clinical trials, this requirement is described in the recent update to ICH E6 (R2) which states, "the sponsor should identify those processes and data that are critical to ensure human subject protection and reliability of trial results." Not all processes in the clinical development landscape have the same level of risk. Therefore, the level of controls to reduce or avoid the risk must be in place and should be commensurate with the level of risk. Processes that pose greater risks have greater levels of control to reduce or avoid these risks. Likewise, processes that pose fewer risks can have fewer controls in place. Actions to reduce process risk include increasing detectability through monitoring, metrics, and controlled documentation. Actions to avoid the risk include removing the source of risk, redesigning a process, and implementing error-proofing techniques. A predetermined and fit-for-purpose risk evaluation framework should be defined and leveraged as part of effective process management. A model risk management framework has been described in TransCelerate's Risk Management Concept Paper. ${ }^{2}$ Applying this framework can ensure consistent identification, assessment, and control of identified process risks.

The level at which a process risk evaluation is conducted also needs to be determined. Generally, process risk evaluation should be completed at a level sufficient to describe the actual 

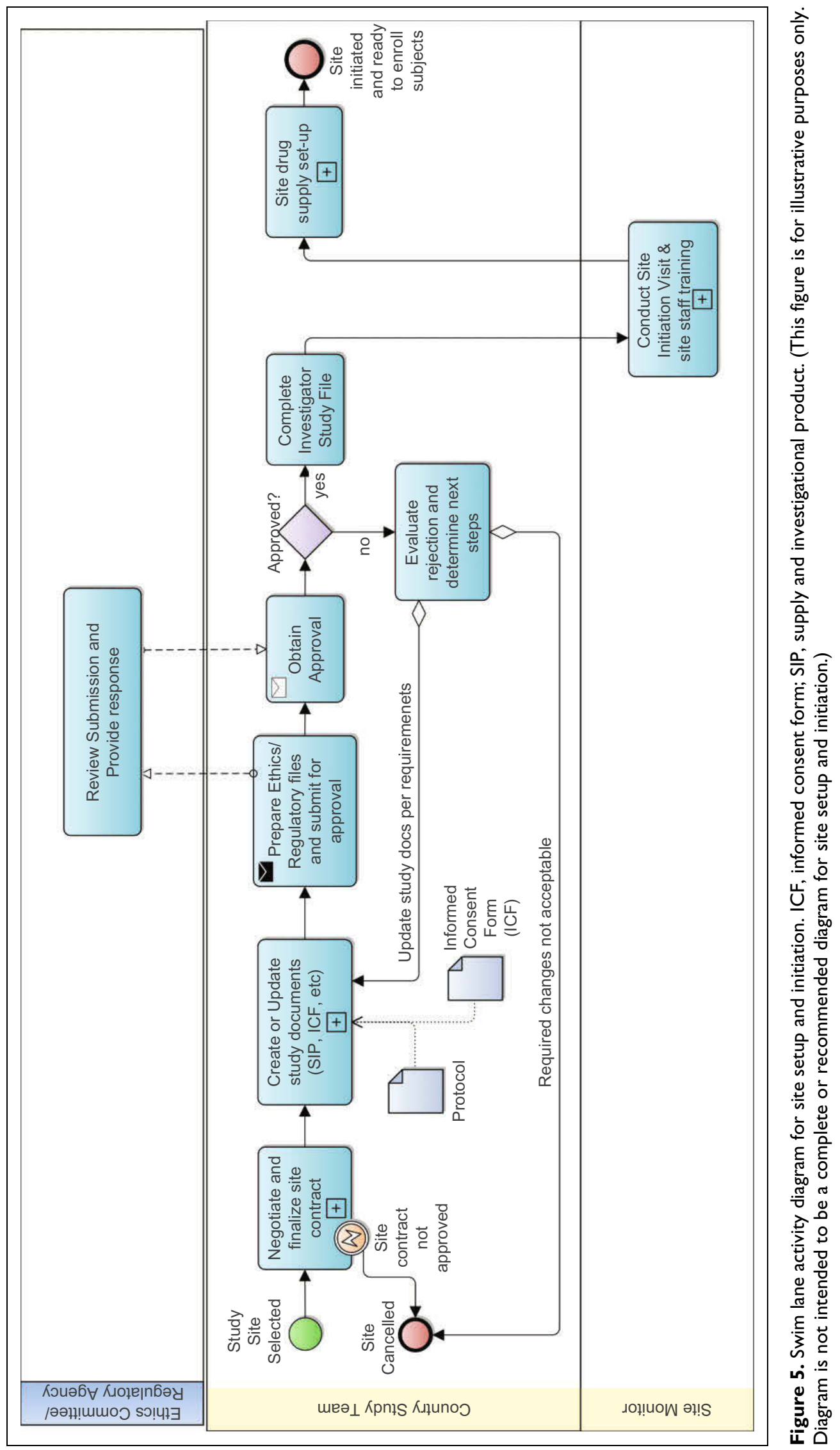
process, roles, handoffs, and activities. Using our example in Figure 5 , there are 5 primary processes or activities that are part of site setup and Initiation. These 5 processes are the following:

- Negotiate and finalize contract

- Create or update study documents

- Prepare, obtain approval, and complete investigator study file

- Conduct site initiation and training

- $\quad$ Site drug supply and setup

Each process needs to be evaluated in terms of process risk. While each of these 5 processes are important to the protection of human subjects, data integrity/reliability of trial results, and quality objectives, the risk evaluation may determine that they are not equal in terms of process risk. Therefore, the action taken (accept, avoid, or reduce) regarding the process risk will be different for each of these processes.

\section{Evaluate Business Process Automation}

The process being documented should be evaluated to determine if the complexities or risks of the process warrant the use of work flow automation to drive consistency of execution. An example of automation is a clinical trial management system (CTMS) that helps manage documentation per site and provides a "green-light" to release investigational products to the site. While not all processes are suited for automation, time- or resource-intensive processes, those prone to human error, and/ or those with higher risk often make good candidates for automating. Organizations should proactively assess processes based on these criteria and strategically identify processes that can benefit from automation.

\section{Identify Process Measures and Controls}

Establishing the correct process performance measures and controls is fundamental and necessary to ensure that the process is working as designed and has objective evidence for evaluating performance and driving continuous improvement. At a minimum, identifying and implementing effective process measures should include (1) a clear definition of what is "critical to quality" (CTQ) from the customer perspective and how to consistently measure it, (2) agreed performance targets, and (3) ownership for the performance of the metric. The SIPOC diagram is a good place to begin to identify effective metrics for a process. Using this as a starting point, one can identify the process outputs and the customers who receive those outputs to define what success looks like and what the process measures and target should be.

Another key concept regarding metrics and driving process improvement is the discussion surrounding primary, lagging, or outcome metrics versus secondary, leading, or causal metrics. Generally primary, lagging, or outcome metrics are those metrics directly associated with the output of the process that is important to the "customer." Secondary, leading, or causal metrics are associated with the input or process activities important or "causal" to the outputs. As a rule of thumb, primary or lagging process measures in clinical development should address time, cost, and quality. Time measures can be less difficult to define (eg, cycle time from when study documentation is completed until regulatory approval is obtained and time from when the first subject is enrolled until the recruitment is completed). These parameters can be measured in terms of costs as well (eg, cost for the company to initiate sites, cost to complete the recruitment for the study, and cost if the recruitment is delayed and additional sites and countries need to be added). Quality measures can be more difficult to measure. Generally quality measures should be associated with "right first time" and conformance to requirements (eg, study documentation completeness, on time reporting to external authorities, and the number of data errors are reviewed as part of quality). Organizational understanding of these key concepts and meeting the challenges associated with metrics are vital to effective process management and continuous improvement.

\section{Determine Process Documentation Strategy}

\section{Begin With Process Map}

By clearly defining and characterizing the process, we are now ready to determine an effective process documentation strategy. An effective documentation strategy ensures that documentation represents the actual process, is easy to understand and follow, has the right level of controls and quality oversight, and is consistent from one process to the next. Determining an effective documentation strategy begins with reviewing the process maps, process risks, and quality objectives and determining which processes require "controlled documentation" versus "managed information."

\section{Identify Processes Requiring Controlled Documentation}

Generally, "the level of controlled documentation for any clinical development process should be commensurate with the risks of the activity and the significance of the activity to achieving quality objectives and meeting stakeholder requirements." Controlled documentation can be defined as explicit (documented) knowledge that is managed in a quality management system (QMS) to support document authoring, approval, and versioning. A QMS is considered both a source of truth that provides a complete picture of the process and a system of record that is used for audit or regulatory reporting purposes. Processes that are characterized as higher risk will require a greater level of "controlled documentation." An example of controlled information/documentation is a standard operating procedure (SOP) containing mandatory information required by regulatory health authorities.

\section{Identify Managed Information Required for Performance}

After the need for controlled documentation has been identified, the processes and activities requiring "managed information" will need to be determined. Managed information 


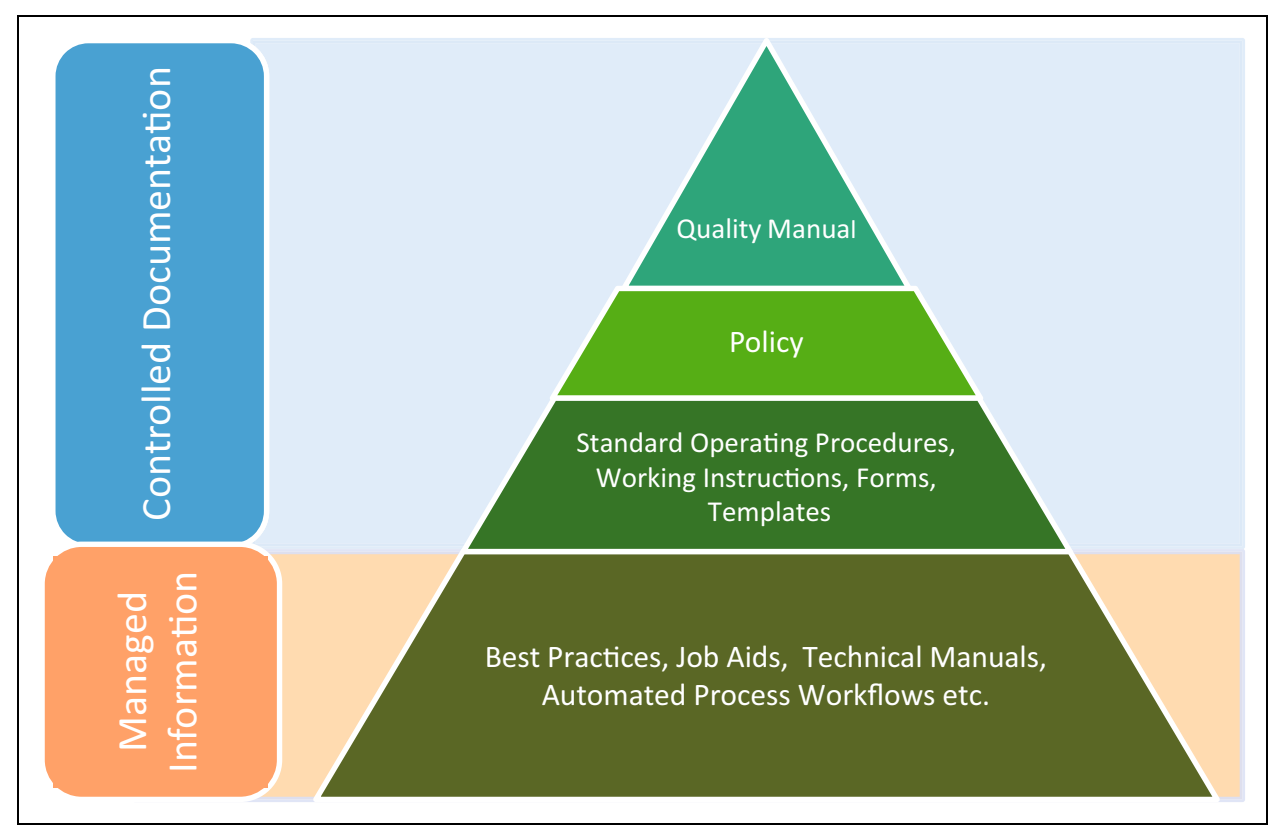

Figure 6. Controlled documentation and managed information hierarchy. Figure is for guidance regarding controlled documentation and managed information. The specific documentation types are for illustrative purposes only. Company-naming conventions may differ.

provides important information and knowledge required to perform the process right first time. Managed information/documentation can be defined as the tacit (experience and insight) knowledge that is not managed in a QMS, but ensures staff has the right information and knowledge needed to execute the process. ${ }^{3}$ An example of this may include materials such as lessons learned that are kept on common company-shared platforms for sharing across study teams.

\section{Align Documentation to E2E Process and Documentation Hierarchy}

It is essential that processes and activities for controlled documentation and managed information are aligned to both the E2E process (eg, study start-up) and the correct documentation hierarchy. This can be a challenging task as existing historical documentation may or may not be aligned. To meet this challenge, consideration should be given to creating new documentation that is aligned to the E2E process and documentation hierarchy versus revising existing documentation. This will require upfront investment; however, this activity is vital to ensure alignment and ownership of the E2E process. To ensure consistent classification of documentation types and alignment to an overall documentation hierarchy, a "documentation hierarchy" should be defined and standardized. An example of this is seen in Figure 6. A few examples of controlled documentation include quality manuals (eg, describes quality management structure and key processes), policies, SOPs, working instructions (WINs), and forms/templates. Some examples of managed information include job aids, guidance documents, technical manuals, automated process workflows, etc.
In clinical development, it is common to have both controlled documentation and managed information for a process. For instance, an SOP (controlled documentation) on study monitoring describes what must be done and who is responsible for it, whereas the "how" aspects might be described/presented in a different format and media (interactive presentations, videos, applications, etc) as best practice solutions (managed information), which are strongly recommended, but allow modifications when there is a business need. It is important that managed information follow good documentation practices.

Connecting staff to controlled documentation and managed information is essential. One way to do this is to create a knowledge hub/center with searchable content. A clinical knowledge management champion/specialist (or similar role) can manage the knowledge hub/center and provide guidance to BPOs regarding the managed information to be developed for their areas. An effective knowledge hub/center "increases productivity by enabling staff to locate and apply available knowledge faster and enhances the quality of clinical development by minimizing repeated mistakes."3 Although a searchable system may not be implemented at all organizations, it is important to be able to align controlled/managed knowledge to the E2E business processes to assess gaps and overlaps.

Using the site set-up and initiation process in Figure 5, the concepts of "controlled" documentation and "managed" information can be applied. For instance, it can be determined that "Create and Update Study Documents" represents a "high-risk" process based on the risk assessment. Therefore, the process would need to have an appropriate level of controlled documentation. An SOP would be needed to document the Create 
and Update Study Documents process. Importantly, based on risk, this process may also require the additional rigor of managed information such as a checklist to ensure requirements are met to drive performance.

\section{Communicate Process Change}

Prior to controlled documentation being assigned to staff, targeted email messages are often used to communicate recently developed or revised documentation for medical, clinical, regulatory, and pharmacovigilance activities to end users. The message may include a list of new/updated documents released and/or cancelled documents, applicability, reasons for creation/ update/cancellation, dates of release/update/cancellation and effective dates, considerations for implementation, and training courses. Additionally, it is recommended that the email communication be stored in an official process documentation repository so that it is easily available to end users implementing the changes over time.

After processes are clearly defined and documents are determined, focused and streamlined training for relevant clinical development processes and associated documentation is key to effective implementation.

\section{Drive Flawless Execution Through Optimal Learning Approach}

\section{Select Optimal Learning Method and Time}

It is important to select the optimal learning delivery based on organizational culture, documentation, knowledge required, experience and target size of audience, and complexity of the process.

There are various learning methods including read and apply, virtual or face-to-face classroom instructor led, e-learning, and video. Depending on the type of process, there may need to be a period of mentoring and on-the-job training before confirming the employee is qualified to independently execute the process. Among the 19 TransCelerate member companies surveyed in 2017, instructor-led training was considered by far the most effective (94\%) followed by e-learning modules (88\%). Read-and-apply training was considered ineffective by more than $50 \%$ of the companies surveyed.

New trends in learning involve gamification (ie, applying gaming designs and concepts to learning or training scenarios), augmented or virtual reality (ie, putting the learner directly in a real or simulated learning environment), and software/technology. Importantly, our world and how people learn and interact with technology continue to evolve, and our industry needs to evaluate and embrace these methods and technologies.

In addition to the supporting technology, consideration should also be given to determining the optimal delivery method and timing of the training. To be most effective, training should be assigned close to the moment of execution (just-in-time training). In a survey of TransCelerate member companies, $70 \%$ of survey respondents already apply $(29 \%)$ or plan to introduce $(41 \%)$ just-in-time training. Although just-intime training drives effectiveness of training, a significant effort is needed from line managers to assign training in line with scheduled activities.

A learning management tool can be anything from an MS Excel matrix to a more automated learning management system (LMS). Learning management tools support organizations in assigning (re-)training to individuals per specified roles, capturing training evidence and training evidence outcomes, and providing oversight on the training status of the workforce.

Ideally, organizations should support learners to learn anything anywhere and from any device. Enabling mobile learning will allow learners to pick up assigned training or pull learning content at any given moment and place.

\section{Select Learning Approach for Controlled and Managed Information}

A learning program consists of 2 elements:

1. Mandatory training based on controlled information (Push): The first element includes learning modules critical for an individual to execute his or her job. At all times, an employee needs to demonstrate effective training was completed prior to executing related tasks.

2. Voluntary training based on managed information (Pull): The second recommended element of learning can be the pulling of learning content as needed. Knowledge centers can be set up for access to SMEs and for the pulling of information. ${ }^{4}$

\section{Manage Training Volume and Avoid Cognitive Overload}

Role-based versus E2E process understanding training Learning modules must allow learners to understand the full process E2E and understand their specific role and responsibilities in that process. To avoid cognitive overload, training for any individual person should focus on the elements related to the person's specific role and an overall understanding of the $\mathrm{E} 2 \mathrm{E}$ process but avoid including detailed training for all roles.

Risk-based training: During onboarding, new employee training is focused on the full understanding of E2E processes. Ongoing learning, after onboarding, is focused on new elements, changes (eg, changes in response to regulatory changes), and high-risk elements of processes. Especially complex tasks or critical deliverables are associated with high risks and require sufficient attention during training.

\section{Delta (differences) training and training waivers}

During onboarding, new employee training is focused on the full understanding of E2E processes; ongoing learning is focused on new elements, changes (delta training), and highrisk elements of processes. When people already have experience (eg, through experience in another function or company), they can be exempted from general parts of training and their onboarding curriculum reduced to company- and/or role- 
specific parts only. The more experience personnel gain while executing company processes, the less the need for standard retraining. While continuing to train on changed or new elements in the processes, proficiency can be tested through knowledge and skills checks against pre-established expected proficiency levels. A successful knowledge check can replace standard retraining of an individual on unchanged processes. Retraining is only required if proficiency is not demonstrated during the knowledge check.

While role-based, risk-based, and delta training focus on managing training volume from a content perspective, the concept of just-in-time training targets training a person at the right moment, avoiding training being assigned that does not relate to the activities scheduled.

Note on process automation: Through automation of processes, the executer (and learner) do not have to read, memorize, or internalize either the sequence of different steps or the criteria for decision making at certain decision points. Criteria for decision making can be built-in, guiding the executer consistently through the same path under the same conditions. While ensuring consistency of execution and reducing execution errors, automation also reduces the need for extensive process training. However, it remains important that learners continue to think critically, do not become too reliant on the automated process, and continue to understand the E2E process.

\section{Monitor and Improve Process Performance}

\section{Evaluate Process Health Performance as Part of Management Review}

Management review is a vital part of the QMS to ensure the QMS is fit-for-purpose and operating as intended. ${ }^{5}$ A central component of management review is engaging senior management in the evaluation of processes to ensure they are fit for purpose and performing as intended so that actions can be taken and resources provided to improve processes as needed. As previously stated, process metrics or key performance indicators (KPIs) need to be established as objective ways to measure quality and compliance health. These metrics should be consistently reviewed and presented by the associated BPOs during management review.

As an example, the completion of corrective and preventive actions (CAPAs) is a fundamental aspect of the QMS and process ownership. Metrics should be established to ensure CAPAs are completed on time and effective in reducing or eliminating recurrence of issues.

\section{Assess Impact of Ongoing Process Changes}

A change or revision to a process should be assessed to ensure the proposed process change is warranted. Before developing processes and procedural documentation to mitigate a risk, the organization should verify that this action will meaningfully reduce the risk or eliminate the root cause. Generally, processes and procedural documentation should be created or modified primarily for the following reasons:

- Address changes in the underlying processes (eg, process improvements)

- Address evolving customer requirements (eg, health authority requirements)

- Address changes identified by audit findings

- Mitigate risks to quality objectives

If the process change is warranted, the organization assesses the impact of the change to accomplish the following:

- Understand the overall impact that the process change may have on other "upstream" or "downstream" processes

- Identify conflicting or overlapping priorities associated with change

- Provide resource and commitment for change projects

\section{Complete Periodic Review of Process}

Periodic review of individual processes should take place as part of a proactive company culture to ensure processes remain fit for purpose and in compliance with regulations. Information from Issue Management (eg, trends in issues) should be evaluated to determine if issues can be alleviated through process redesign and/or the development, revision, or retirement of specific procedural documents. Knowledge management and risk management activities may also identify changes that warrant reevaluation of processes and associated documents that could be retired, simplifying the procedural documentation infrastructure. In addition, process performance metrics and trends should be evaluated. The periodic review of processes should occur on a predetermined schedule (eg, every year or every 3 years) depending on the process.

\section{Conclusions}

The process management framework described herein represents an integral part of an effective QMS. Common understanding of the steps required to implement this framework can provide greater organizational success in implementing process management in a robust way. Successful implementation of the process management framework will ensure key and critical processes associated with clinical development are identified, defined, and have clear and concise procedural documentation that is consistent and fit for purpose. It will also ensure that staff are effectively trained and able to excel at executing the process "right first time." This is a "must have" for today's competitive landscape. To further aid in implementation of the process management framework and concepts outlined in this manuscript, an accompanying "implementation toolkit" that provides tools and important examples for each step of the framework have been developed. These tools will be available on the TransCelerate webpage at www.transceleratebiopharmainc.com. 


\section{Acknowledgments}

All authors were involved in the writing and editing of the article. As applicable, licenses for all figures have been purchased.

The authors gratefully acknowledge the support of TransCelerate BioPharma Inc, a nonprofit organization dedicated to improving the health of people around the world by accelerating and simplifying the research and development (R\&D) of innovative new therapies. The organization's mission is to collaborate across the global biopharmaceutical R\&D community to identify, prioritize, design, and facilitate implementation of solutions designed to drive the efficient, effective, and high-quality delivery of new medicines.

The authors also gratefully acknowledge the support of the following TransCelerate working team members who contributed to the concepts described in this manuscript: Liqi Chen, MD, MBA, Astellas; Mary Fleres Allergan; Milena Santos, MS, Merck/MSD; Anette Stenson, MSc, Astra Zeneca; Sandra Fuelbier, BS, EMD, Serono.

\section{Declaration of Conflicting Interests}

No potential conflicts were declared.

\section{Funding}

No financial support of the research, authorship, and/or publication of this article was declared.

\section{ORCID iD}

Michael Edward Husovich, BS (D) https://orcid.org/0000-0001-73099628

\section{References}

1. Meeker-O'Connell A, Borda MM, Little JA, Sam LM. Enhancing quality and efficiency in clinical development through a clinical QMS conceptual framework: concept paper vision and outline. Therapeutic Innovation \& Regulatory Science. 2015;49(5): 615-622.

2. Suprin, M, Chow A, Pillwein M, et al. Quality risk management framework: guidance for successful implementation of risk management in clinical development. Therapeutic Innovation \& Regulatory Science. 2018; 1-9; DOI: 10.1177/2168479018817752.

3. Salzano KA, Maurer CA, Wyvratt JM, et al. A knowledge management framework and approach for clinical development. Therapeutic Innovation \& Regulatory Science. 2016;50(5):536-545.

4. van Merrienboer JJG, Clark RE, de Croock MBM. Blueprints for complex learning: the 4C/ID-model. ETR\&D. 2002;50(2):39-64.

5. Meeker-O'Connell A, Sam LM, Bergamo N, Little JA. TransCelerate's clinical quality management system: from a vision to a conceptual framework. Therapeutic Innovation \& Regulatory Science. 2016;50(4):397-413. 